\title{
Elliptic flow of deuterons in relativistic heavy-ion collisions
}

\author{
Yongseok $\mathrm{Oh}^{*}$ and Che Ming $\mathrm{Ko}^{\dagger}$ \\ Cyclotron Institute and Physics Department, Texas A\&M University, College Station, Texas 77843-3366, USA
}

(Received 31 July 2007; revised manuscript received 18 October 2007; published 28 November 2007)

\begin{abstract}
Using a dynamical model based on the $N N \rightarrow d \pi, N N N \rightarrow d N$, and $N N \pi \rightarrow d \pi$ reactions and measured proton and pion transverse momentum spectra and elliptic flows, we study the production of deuterons and their elliptic flow in heavy-ion collisions at RHIC. The results are compared with those from the coalescence model. The deviation of deuteron elliptic flow from the constituent nucleon number scaling expected from the coalescence model and the comparison with the experimental data are discussed in connection to the allowed nucleon phase space in these reactions.
\end{abstract}

DOI: 10.1103/PhysRevC.76.054910

PACS number(s): 25.75.Ld, 25.75.Dw, 13.75.Cs

\section{INTRODUCTION}

The elliptic flow of particles in heavy-ion collisions is a measure of the strength of the second Fourier coefficient in the azimuthal angle distribution of particle transverse momentum relative to the reaction plane [1,2]. Significant information on the properties of the hot dense matter formed during the initial stage of heavy-ion collisions has been obtained from the study of elliptic flow [3-5]. In heavy-ion collisions at the Relativistic Heavy Ion Collider (RHIC) [6,7], measured elliptic flow at low transverse momentum shows a mass ordering, i.e., the strength of elliptic flow of identified hadrons decreases with increasing hadron masses, and this has been well described by ideal hydrodynamics [8] as well as by the transport model [9]. Furthermore, the elliptic flows of identified hadrons, particularly at intermediate transverse momenta, are seen to follow a constituent quark number scaling, i.e., the dependence of hadron elliptic flows on hadron transverse momentum becomes similar if both are divided by the number of constituent quarks in a hadron. This scaling behavior of hadron elliptic flows is consistent with the predictions of the quark coalescence model for hadron production from produced quark-gluon plasma in relativistic heavy-ion collisions [10-14].

Recently, the elliptic flow of deuteron has also been measured at RHIC [15-17]. These measurements show that the deuteron elliptic flow seems to scale with its constituent nucleon number, implying that the quark number scaling of elliptic flows holds not only for hadrons but also for the deuteron. Because deuteron production in heavy-ion collisions could be described by the coalescence of protons and neutrons at freeze-out [18], the observed nucleon number scaling of the deuteron elliptic flow is thus not surprising. In the simplest coalescence model $[13,14]$ which involves only comoving particles, the deuteron yield in momentum space is proportional to the product of the proton and neutron densities at half the momentum of produced deuteron, i.e., $\boldsymbol{p}_{p}=\boldsymbol{p}_{n}=\boldsymbol{p}_{d} / 2$, and the deuteron elliptic flow would satisfy exactly the nucleon number scaling and thus the quark number

\footnotetext{
*yoh@comp.tamu.edu

${ }^{\dagger}$ ko@comp.tamu.edu
}

scaling as well. This strong momentum constraint is relaxed in the more general coalescence model [18] that takes into account the nucleon momentum spread in the deuteron. As in the case of hadron production from quark coalescence [10], the more general coalescence model would lead to a small deviation of the scaled deuteron elliptic flow from that of nucleons.

Although the momentum conservation is maintained in the coalescence model, the energy conservation is not satisfied. Some doubt has thus been raised on the applicability of the coalescence model, especially in low transverse momentum region $[19,20]$. Investigating the consequences arising from the energy conservation condition is therefore required to test the coalescence model and to understand the constituent number scaling of elliptic flows in the low-transverse-momentum region. In this work, this question will be addressed by studying deuteron production and elliptic flow in heavy-ion collisions based on a dynamical approach that satisfies both energy and momentum conservations. Specifically, deuteron production in the present study will be treated through $N N \rightarrow$ $d \pi, N N N \rightarrow d N$, and $N N \pi \rightarrow d \pi$ reactions. Because this approach is based on physical scattering processes for deuteron production, the energy-momentum conservation is always satisfied. By comparing the physically allowed nucleon phase space for deuteron production with that involved in the coalescence model, we can study the consequences of the energy conservation condition. In a realistic study, these reactions need to be included in the hadronic stage of relativistic heavyion collisions either via a microscopic transport model [21] or a schematic kinetic model [22] to take into account all possible deuteron production and dissociation processes. Because the elliptic flow of particles during the hadronic evolution does not change much as the initial spatial asymmetry through which the elliptic flow is generated has decreased significantly during the evolution of the partonic stage [9], we can therefore calculate the deuteron yield and elliptic flow based on the deuteron production rate from nucleons at freeze-out.

This article is organized as follows. In Sec. II, we first give a brief description of the coalescence model that will be used to compare with the results from the dynamical model employed in the present study for deuteron production. We then consider in Sec. III and Sec. IV deuteron production from the two-body reaction $N N \rightarrow d \pi$ and three-body reactions $N N N \rightarrow d N$ 
and $N N \pi \rightarrow d \pi$, respectively, with their cross sections obtained from a hadronic model that is based on empirically determined parameters [23]. In Sec. V, nucleon and pion transverse-momentum spectra and elliptic flows, which are needed in both the coalescence model and the dynamical model for studying deuteron production, are discussed. Results on deuteron transverse-momentum spectrum and elliptic flow are then compared with available experimental data from RHIC as well as those from the coalescence model in Sec. VI, where we further compare the nucleon phase space involved in our dynamical model with that in the coalescence model. Section VII contains the summary and discussions. Details on the reaction amplitudes for both two-body and three-body reactions are given in Appendixes $\mathrm{A}$ and $\mathrm{B}$, respectively.

\section{DEUTERON PRODUCTION IN THE COALESCENCE MODEL}

In the coalescence model, the probability for the production of a bound composite particle from a many-particle system is determined by the overlap of the wave functions of coalescing particles with the internal wave function of the composite particle [24]. Assuming that nucleons are uniformly distributed in space, the momentum spectrum of deuterons formed from the coalescence of nucleons is given by

$$
\begin{aligned}
\frac{d N_{d}}{d^{3} \boldsymbol{p}_{d}}= & \frac{3}{4} \frac{V}{(2 \pi)^{3}} \int d^{3} \boldsymbol{p}_{1} d^{3} \boldsymbol{p}_{2} f_{p}\left(\boldsymbol{p}_{1}\right) f_{n}\left(\boldsymbol{p}_{2}\right) \\
& \times\left|\Psi_{d}\left[\left(\boldsymbol{p}_{1}^{\prime}-\boldsymbol{p}_{2}^{\prime}\right) / 2\right]\right|^{2} \delta^{(3)}\left(\boldsymbol{p}_{1}+\boldsymbol{p}_{2}-\boldsymbol{p}_{d}\right) .
\end{aligned}
$$

In the above, the factor $3 / 4$ is the probability for a proton and a neutron to form a spin triplet state like that in a deuteron; $f_{p}\left(\boldsymbol{p}_{1}\right)$ and $f_{n}\left(\boldsymbol{p}_{2}\right)$ are, respectively, the proton and neutron momentum distributions in the hadronic matter of volume $V$ at freeze-out and they are related to the proton number $N_{p}$ and neutron number $N_{n}$ by $V \int \frac{d^{3} \boldsymbol{p}}{(2 \pi)^{3}} f_{p, n}(\boldsymbol{p})=N_{p, n} ; \Psi_{d}$ is the deuteron wave function in the momentum space with $\boldsymbol{p}_{1}^{\prime}$ and $\boldsymbol{p}_{2}^{\prime}$ denoting, respectively, the momenta of the proton and neutron in the deuteron rest frame. In the more general case of nonuniform nucleon distribution and/or with collective flow, additional spatial integrals would appear in Eq. (1), and the deuteron momentum space wave function is replaced by its Wigner function, which describes both the relative momentum and spatial distributions of the two nucleons in a deuteron $[25,26]$.

For the deuteron wave function, we use the one given by Hulthén. In the momentum space, it is given by

$$
\Psi_{d}(k)=\frac{\sqrt{\left(\alpha_{d}+\beta_{d}\right)^{3} \alpha_{d} \beta_{d}}}{\pi\left(\alpha_{d}^{2}+k^{2}\right)\left(\beta_{d}^{2}+k^{2}\right)},
$$

with $\alpha_{d}=0.23 \mathrm{fm}^{-1}$ and $\beta_{d}=1.61 \mathrm{fm}^{-1}$ [26] and is normalized as $\int d^{3} \boldsymbol{k}\left|\Psi_{d}(k)\right|^{2}=1$. It should be mentioned that this more general coalescence model still does not respect the energy conservation condition.

In the naive coalescence model, the deuteron wave function in the momentum space is taken to be a $\delta$ function. In this case, the transverse momenta $\boldsymbol{p}_{T, 1}$ and $\boldsymbol{p}_{T, 2}$ of the nucleons forming the deuteron are restricted not only in magnitude but also in direction so that $\boldsymbol{p}_{T, 1}=\boldsymbol{p}_{T, 2}=\boldsymbol{p}_{T, d} / 2$ with $\boldsymbol{p}_{T, d}$ being the deuteron transverse momentum $[13,14]$. As a result, the scaling of the nucleon elliptic flow $v_{2, N}$ and the deuteron elliptic flow $v_{2, d}$ according to their constituent nucleon numbers is exact, i.e., $v_{2, d}\left(p_{T}\right)=2 v_{2, N}\left(p_{T} / 2\right)[14,27]$.

\section{DEUTERON PRODUCTION FROM TWO-BODY REACTIONS}

For deuteron production from two-body nucleon-nucleon reactions, the dominant reaction is $N N \rightarrow d \pi$ due to the small pion mass and the strong pion-nucleon coupling. Indeed, experimental observations have shown that the cross section for the $N N \rightarrow d \pi$ reaction is much larger than the cross sections for deuteron production from other two-body reactions [23]. In contrast to the coalescence model, in which the deuteron is formed from a proton and a neutron in the hadronic matter, not only the $p n$ reaction but also the $p p$ and $n n$ reactions can produce a deuteron in the $N N \rightarrow d \pi$ reaction as the pion, which has isospin one, can carry away the mismatched isospin.

The production rate of deuterons with momentum $\boldsymbol{p}_{d}$ from the $N N \rightarrow d \pi$ reaction can be written as

$$
\begin{aligned}
\frac{d \mathcal{R}_{d}}{d^{3} \boldsymbol{p}_{d}}= & \frac{1}{(2 \pi)^{3} 2 E_{d}} \int \prod_{i=1}^{2} \frac{d^{3} \boldsymbol{p}_{i}}{(2 \pi)^{3} 2 E_{i}} f_{N}\left(\boldsymbol{p}_{i}\right) \frac{d^{3} \boldsymbol{p}_{\pi}}{(2 \pi)^{3} 2 E_{\pi}} \\
& \times\left[1+f_{\pi}\left(\boldsymbol{p}_{\pi}\right)\right]|\mathcal{M}(N N \rightarrow d \pi)|^{2} \\
& \times(2 \pi)^{4} \delta^{(4)}\left(\sum_{i=1}^{2} p_{i}-p_{\pi}-p_{d}\right),
\end{aligned}
$$

where $f_{N}(\boldsymbol{p})$ and $f_{\pi}\left(\boldsymbol{p}_{\pi}\right)$ are nucleon and pion momentum distribution functions with the latter normalized to the pion number in the hadronic matter. We have assumed here that the proton and neutron densities in the hadronic matter are the same for simplicity. The average of initial spin and isospin states and sum over final spin and isospin states are included in the squared transition amplitude $|\mathcal{M}(N N \rightarrow d \pi)|^{2}$. Details on the amplitude for the $N N \rightarrow d \pi$ reaction are given in Appendix A. Compared with the coalescence model, Eq. (3) shows that both energy and momentum are always conserved due to the produced pion. This allows nucleons with higher momentum to produce a low-momentum deuteron because the pion can carry off the excess energy of the two nucleons. As a result, there is no a priori restriction on the momenta such as $p_{T, d} \approx 2 p_{T, N}$ as in the coalescence model. Instead, the nucleon distribution and the energy-momentum conservation condition determine the most plausible configurations of nucleon momentum for producing the deuteron.

\section{DEUTERON PRODUCTION FROM THREE-BODY REACTIONS}

Deuterons can also be produced from three-body reactions such as $N N N \rightarrow d N$ and $N N \pi \rightarrow d \pi$. The reaction $N N N \rightarrow d N$ is known to dominate deuteron production in low-energy heavy-ion collisions [28,29]. Because of the large abundance of pions in the produced hadronic matter 
in heavy-ion collisions at RHIC, the reaction $N N \pi \rightarrow d \pi$ becomes also possible.

\section{A. $N N N \rightarrow d N$ reaction}

For the $N N N \rightarrow d N$ reaction, the production rate for deuterons of momentum $\boldsymbol{p}_{d}$ is given by

$$
\begin{aligned}
\frac{d \mathcal{R}_{d}}{d^{3} \boldsymbol{p}_{d}}= & \frac{1}{(2 \pi)^{3} 2 E_{d}} \int \prod_{i=1}^{3} \frac{d^{3} \boldsymbol{p}_{i}}{(2 \pi)^{3} 2 E_{i}} f_{N}\left(\boldsymbol{p}_{i}\right) \frac{d^{3} \boldsymbol{p}_{N}}{(2 \pi)^{3} 2 E_{N}} \\
& \times\left[1-f_{N}\left(\boldsymbol{p}_{N}\right)\right]|\mathcal{M}(N N N \rightarrow d N)|^{2} \\
& \times(2 \pi)^{4} \delta^{(4)}\left(\sum_{i=1}^{3} p_{i}-p_{N}-p_{d}\right) .
\end{aligned}
$$

The presence of the third nucleon in the initial state leads to a very different nucleon phase space in deuteron formation, as the third (spectator) nucleon can ensure the energy-momentum conservation. Because the nucleon mass is much larger than the pion mass, the effect of the spectator nucleon is different from that of the pion produced in the $N N \rightarrow d \pi$ reaction and thus could affect the elliptic flow of produced deuteron differently. The amplitude for the $N N N \rightarrow d N$ reaction can be obtained from its inverse reaction $d N \rightarrow N N N$, which has been studied in Refs. [29-31] based on the full $N N$ elasticscattering amplitude. Instead of such a complete study, we approximate the amplitude for the reaction $N N N \rightarrow d N$ by extending the amplitude we derived in the previous section for the $N N \rightarrow d \pi$ reaction to an off-shell pion that is absorbed by the third spectator nucleon. Details on the modeling of the amplitude $\mathcal{M}(N N N \rightarrow d N)$ are given in Appendix B.

\section{B. $N N \pi \rightarrow d \pi$ reaction}

The same method introduced in the above for the $N N N \rightarrow$ $d N$ reaction can be used for the amplitude of the $N N \pi \rightarrow d \pi$ reaction, i.e., the subprocess $N N \rightarrow d \pi$ is replaced by $N N \rightarrow$ $d \rho$ with the off-shell $\rho$ meson absorbed by the spectator pion. Details on the modeling of the amplitude $\mathcal{M}(N N \pi \rightarrow d \pi)$ are also given in Appendix B. In terms of the nucleon and pion momentum distributions the production rate for deuterons with momentum $\boldsymbol{p}_{d}$ from this reaction is given by

$$
\begin{aligned}
\frac{d \mathcal{R}_{d}}{d^{3} \boldsymbol{p}_{d}}= & \frac{1}{(2 \pi)^{3} 2 E_{d}} \int \prod_{i=1}^{2} \frac{d^{3} \boldsymbol{p}_{i}}{(2 \pi)^{3} 2 E_{i}} f_{N}\left(\boldsymbol{p}_{i}\right) \frac{d^{3} \boldsymbol{p}_{3}}{(2 \pi)^{3} 2 E_{3}} \\
& \times f_{\pi}\left(\boldsymbol{p}_{3}\right) \frac{d^{3} \boldsymbol{p}_{\pi}}{(2 \pi)^{3} 2 E_{\pi}}\left[1+f_{\pi}\left(\boldsymbol{p}_{\pi}\right)\right] \\
& \times|\mathcal{M}(N N \pi \rightarrow d \pi)|^{2} \\
& \times(2 \pi)^{4} \delta^{(4)}\left(\sum_{i=1}^{3} p_{i}-p_{\pi}-p_{d}\right) .
\end{aligned}
$$

\section{NUCLEON AND PION TRANSVERSE-MOMENTUM DISTRIBUTIONS}

Both the coalescence model and the dynamical model use as input the nucleon momentum distribution at freeze-out Moreover, the pion momentum distribution at freeze-out is also needed in the dynamical model. For heavy-ion collisions at RHIC, both nucleon and pion rapidity distributions are approximately uniform in the midrapidity region, i.e., $|y| \leqslant 0.5$, which we will assume in the present study. In this case, only their transverse-momentum distributions are needed. In the following, we discuss how these distributions are obtained.

\section{A. Nucleon spectrum and elliptic flow}

For the nucleon $p_{T}$ distribution $f_{N}\left(\boldsymbol{p}_{T}\right)$, we take it to have the form

$$
f_{N}\left(\boldsymbol{p}_{T}\right)=\gamma_{N} \exp \left(-m_{T} / T_{\text {eff }}\right)\left[1+v_{2, N}\left(p_{T}\right) \cos (2 \phi)\right],
$$

where $m_{T}=\sqrt{M_{N}^{2}+p_{T}^{2}}$ with the nucleon mass $M_{N}$ and $\phi$ being the azimuthal angle of the nucleon transverse momentum with respect to the reaction plane. We have introduced a dimensionless parameter $\gamma_{N}$ to compensate for the effects caused by using the effective inverse slope parameter $T_{\text {eff }}$ instead of the temperature of the system together with the radial flow effect. The parameter $\gamma_{N}$ also contains the density of spin and isospin states as well as the possible effect due to finite nucleon chemical potential.

The inverse slope parameter in the nucleon $p_{T}$ spectrum [Eq. (6)] is determined to be $T_{\text {eff }}=295 \mathrm{MeV}$ by using the experimental data of Ref. [32] for the minimum bias proton $p_{T}$ spectrum. Taking the effective volume of the hadronic matter at freeze-out in minimum bias collisions to be $V \approx 3,700 \mathrm{fm}^{3}$, which is about $1 / 3$ of the estimated hadronic matter volume in central Au+Au collisions at $\sqrt{s_{N N}}=200 \mathrm{GeV}$ [22], we obtain $\gamma_{N}=0.021$ for the value of the parameter in Eq. (6). ${ }^{1}$ The comparison of our parameterized proton $p_{T}$ spectrum, which is half of the nucleon $p_{T}$ spectrum, with the experimental data is shown in Fig. 1(a).

For the nucleon elliptic flow $v_{2, N}$, we assume that it is the same for both protons and neutrons and parametrize it in the form of the Fisher-Tippet (or Gumbel) distribution function [33], i.e.,

$$
v_{2, N}\left(p_{T}\right)=\alpha_{N} \exp \left\{-\exp \left[\left(\lambda_{N}-p_{T}\right) / \beta_{N}\right]\right\} .
$$

We use the experimental data of Ref. [16] for the proton $v_{2}$, which were measured for $20-60 \%$ centrality, to fix the parameters. This gives $\alpha_{N}=0.258, \beta_{N}=0.683 \mathrm{GeV}$, and $\lambda_{N}=1.128 \mathrm{GeV}$. This parametrization is shown in Fig. 1(b) together with the experimental data of Ref. [16]. Our parametrization gives similar results in the measured $p_{T}$ region as the parametrization $a \tanh \left(b p_{T}+c\right)$, which was introduced in Ref. [34] for quarks. But using the latter form would lead to a negative $v_{2}$ at small $p_{T}$. Although there is no experimental information on the nucleon $v_{2}$ at very low $p_{T}$ region, hydrodynamic model studies $[35,36]$ show that the nucleon $v_{2}$ produced in heavy-ion collisions at RHIC has non-negative values [5]. Because the hydrodynamic

\footnotetext{
${ }^{1}$ The difference of the proton $p_{T}$ spectrum in minimum bias and in central collisions shows that the volume of the hadronic matter in the minimum bias collision is about one-third of that in the central collision when the data are parametrized using Eq. (6).
} 

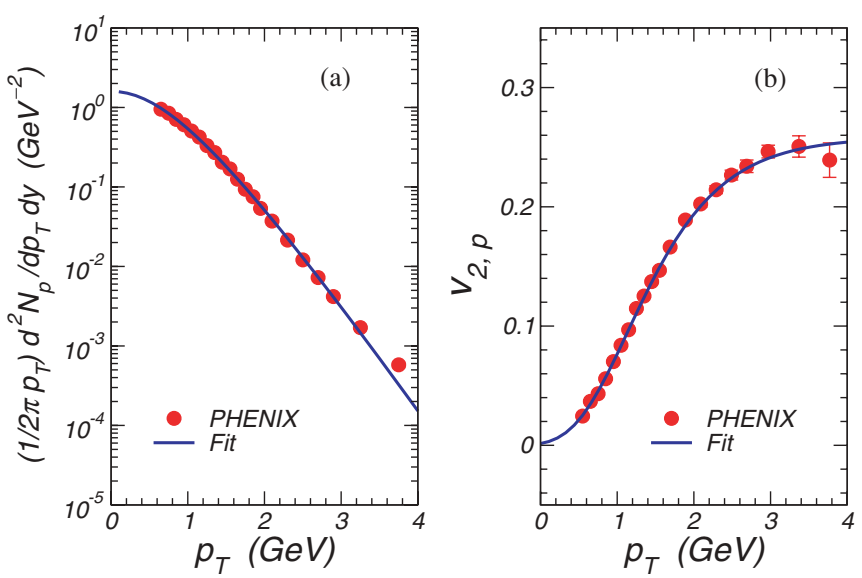

FIG. 1. (Color online) (a) $p_{T}$ spectrum of protons. The solid line is half of the parameterized nucleon distribution [Eq. (6)]. Experimental data are from Ref. [32] for the minimum-bias proton spectrum (filled square). (b) Nucleon differential elliptic flow $v_{2}\left(p_{T}\right)$. The solid line is the parameterized one [Eq. (7)] and filled squares are experimental data from Ref. [16].

calculations are expected to reasonably describe $v_{2}$ at low $p_{T}$ region, we employ the form of Eq. (7) which gives non-negative $v_{2, N}$ even for very small $p_{T}$. We may also take the proton $v_{2}$ to have the form $\alpha^{\prime} p_{T}+\beta^{\prime} p_{T}^{3}$ for $p_{T} \leqslant p_{T}^{0}$ and $a \tanh \left(b p_{T}+c\right)$ for $p_{T} \geqslant p_{T}^{0}$, which gives a similar result as Eq. (7) if we choose $p_{T}^{0}=0.75 \mathrm{GeV}, a=0.293, b=$ $0.460 \mathrm{GeV}^{-1}, c=-0.175, \alpha^{\prime}=0.0332 \mathrm{GeV}^{-1}$, and $\beta^{\prime}=$ $0.0579 \mathrm{GeV}^{-3}$.

\section{B. Pion spectrum and elliptic flow}

For the pion $p_{T}$ spectrum, the parametrization used for the nucleon $p_{T}$ spectrum [Eq. (6)] does not work well as it is affected by resonance decays [34,37]. Instead, we use the parametrization,

$$
f_{\pi}\left(\boldsymbol{p}_{T}\right)=\gamma_{\pi}\left(1+\frac{p_{T}}{\alpha_{\pi}}\right)^{\beta_{\pi}}\left[1+v_{2, \pi}\left(p_{T}\right) \cos (2 \phi)\right],
$$

with $\alpha_{\pi}=1.29 \mathrm{GeV}, \beta_{\pi}=-12.0$, and $\gamma_{\pi}=2.0$ determined from the experimental data of the PHENIX Collaboration [32]. For the pion $v_{2}$, it is parameterized as in Eq. (7),

$$
v_{2, \pi}\left(p_{T}\right)=\alpha_{\pi} \exp \left\{-\exp \left[\left(\lambda_{\pi}-p_{T}\right) / \beta_{\pi}\right]\right\},
$$

with $\alpha_{\pi}=0.184, \beta_{\pi}=0.461 \mathrm{GeV}$, and $\lambda_{\pi}=0.547 \mathrm{GeV}$ to fit the PHENIX experimental data [15]. The comparison of our parametrization with experimental data is shown in Fig. 2.

\section{RESULTS}

With the above proton and pion $p_{T}$ spectra and elliptic flows as inputs, we calculate in this section the deuteron $p_{T}$ spectrum and $v_{2}$ from the dynamical model and compare the results with experimental data as well as those from the coalescence model.
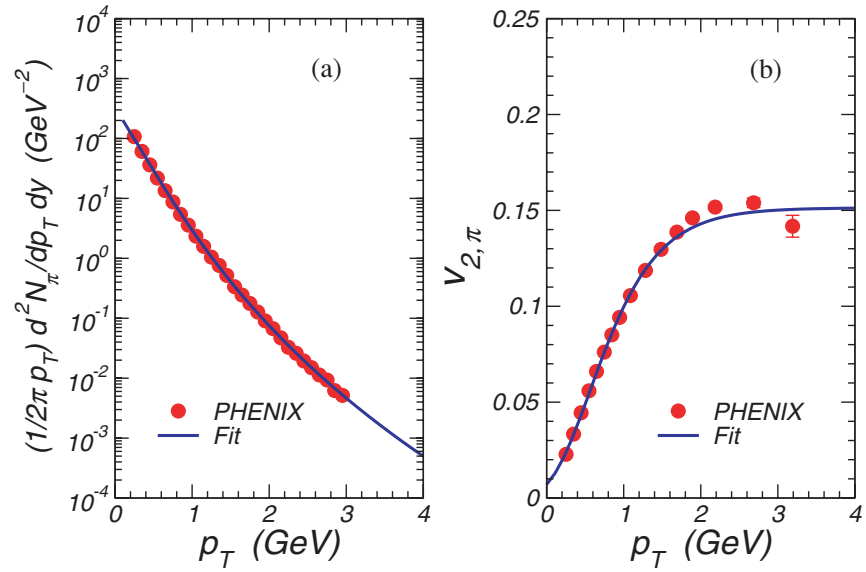

FIG. 2. (Color online) $p_{T}$ spectrum (a) and $v_{2}$ (b) of pions. Solid lines are parametrization of Eqs. (8) and (9). Experimental data are from Refs. [15,32].

\section{A. Deuteron spectrum and elliptic flow from two-body reactions}

In terms of rapidity and transverse momentum, Eq. (3) for the deuteron production rate can be written as

$$
\begin{aligned}
\frac{d^{3} \mathcal{R}_{d}}{d^{2} \boldsymbol{p}_{T, d} d y_{d}}= & \frac{1}{8(2 \pi)^{8}} \int \prod_{i=1}^{2} d y_{i} d p_{T, i} d \phi_{i} p_{T, i} f_{N}\left(\boldsymbol{p}_{T, i}\right) \\
& \times\left[1+f_{\pi}\left(\boldsymbol{p}_{1}+\boldsymbol{p}_{2}-\boldsymbol{p}_{d}\right)\right]|\mathcal{M}(N N \rightarrow d \pi)|^{2} \\
& \times \delta\left[\left(p_{1}+p_{2}-p_{d}\right)^{2}-M_{\pi}^{2}\right] .
\end{aligned}
$$

The deuteron $p_{T}$ spectrum is then obtained by multiplying the above rate by the volume of the hadronic matter at freeze-out $V \approx 3,700 \mathrm{fm}^{3}$ and the time interval $\Delta \tau \approx 4 \mathrm{fm} / c$. The latter value is chosen to reproduce reasonably measured deuteron spectrum. It takes into account the fact that in a more microscopic approach that follows the production and dissociation of deuterons from the reaction $N N \rightarrow d \pi$ as well as the deuteron elastic scattering, deuterons are expected to be produced over a finite time interval from the expanding hadronic matter formed in heavy-ion collisions. To extract the elliptic flow $v_{2}$ of produced deuterons, we further express the result as

$$
\begin{aligned}
\frac{d^{3} N_{d}}{p_{T, d} d p_{T, d} d \Phi_{d} d y_{d}}= & f_{d}\left(p_{T, d}, y_{d}\right) \\
& \times\left[1+2 v_{2, d}\left(p_{T, d}\right) \cos \left(2 \Phi_{d}\right)\right],
\end{aligned}
$$

where $\Phi_{d}$ is the azimuthal angle of produced deuteron and $\phi_{1,2}$ are those of the two nucleons relative to the reaction plane. Because of the $\delta$ function that comes from the energy-momentum conservation and the pion on-shell condition, one of the nucleon azimuthal angles is constrained by kinematics. This is different from the assumption in the naive coalescence model that the direction of the nucleon momentum is constrained to $\phi_{1}=\phi_{2}=\Phi_{d}$.

The resulting deuteron $p_{T}$ spectrum using the phenomenological scattering amplitude given in Appendix A for the $N N \rightarrow d \pi$ reaction is shown by the solid line in Fig. 3(a). Compared with the measured $p_{T}$ spectrum of deuterons shown by filled circles [38], ours is softer. This result indicates 

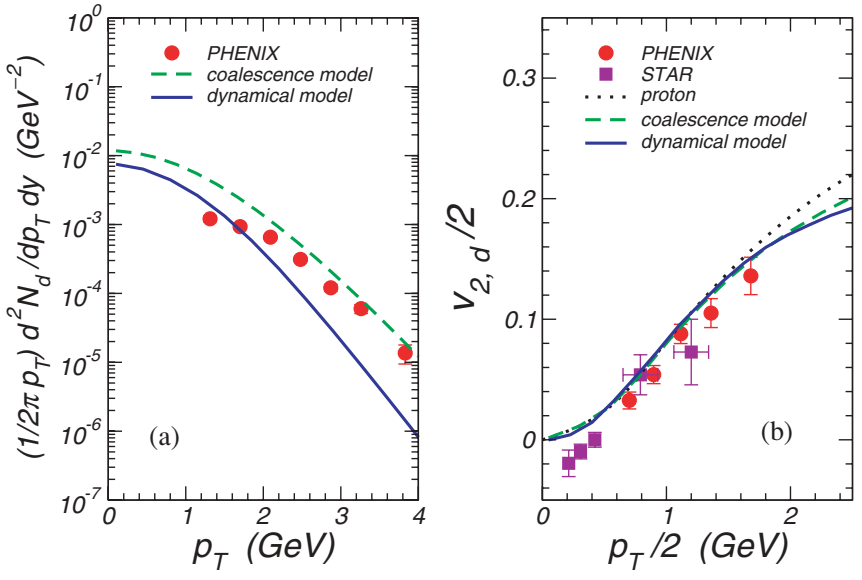

FIG. 3. (Color online) (a) $p_{T}$ spectrum of deuterons. Experimental data are from Ref. [38] for the minimum-bias collisions (filled circle). (b) Scaled deuteron elliptic flow $v_{2}$ as a function of $p_{T} / 2$ at $y_{d}=0$. The dotted line is the nucleon elliptic flow $v_{2, N}$ of Eq. (7). Experimental data are from the PHENIX Collaboration [16] (filled circles) and the STAR Collaboration [17] (filled squares). In both figures, the dashed and solid lines are results from the coalescence model of Eq. (1) and the dynamical model in the present work, respectively.

that the radial flow effect on produced deuterons is not fully taken into account in this dynamical model. For nucleons that can be described by a local thermal distribution modified by radial flow in the fireball frame, we should use the nucleon $p_{T}$ spectrum without radial flow to evaluate the deuteron production rate and then boost resulting deuteron $p_{T}$ spectrum by the radial flow. Also, because the cross section for $\pi d$ elastic and inelastic scatterings are comparable at pion kinetic energy of around $100 \mathrm{MeV}$ [39], deuterons produced from the dynamic model are expected to suffer further scatterings and thus to approach thermal equilibrium with pions. To properly treat the above effects on produced deuteron spectrum requires the inclusion of the processes considered in present study in a nonequilibrium transport model, such as the ART model [40]. As we are mainly interested in studying the effect of energy conservation on the deuteron elliptic flow, we will leave such more realistic approach to a future study. Surprisingly, the deuteron $p_{T}$ spectrum obtained from Eq. (1) of the coalescence model agrees better with the data as shown by the dashed line.

Results from the dynamical model [Eq. (1)] for the scaled deuteron $v_{2}$, i.e., $v_{2, d} / 2$, are shown by the solid line in Fig. 3(b) as a function of $p_{T} / 2$. Compared to the experimental data shown by filled circles from the PHENIX Collaboration and by filled squares from the STAR Collaboration, our model can describe both reasonably well, particularly those from the PHENIX Collaboration [16]. The calculated deuteron $v_{2}$ is, however, always positive, which is not consistent with the negative values of deuteron $v_{2}$ at small $p_{T}$ seen in the preliminary STAR data [17]. This may again be related to our incomplete treatment of radial flow effect and neglect of finalstate interactions of produced deuterons. If the nucleon number scaling of deuteron $v_{2}$ is exact as in the naive coalescence model, the scaled deuteron elliptic flow $v_{2, d}\left(p_{T} / 2\right) / 2$ should be identical to the nucleon elliptic flow $v_{2, N}\left(p_{T}\right)$ [Eq. (7)] shown by the dotted line. Although some deviations are found between the two curves at large $p_{T}$ region, our result supports the idea of the nucleon number scaling of $v_{2, d}$. Furthermore, our result is very similar to that of the more general coalescence model for deuteron production (dashed lines). In the considered energy region, the two approaches give nearly the same $v_{2, d}$. This may indicate that the momentum conservation in the coalescence model plays a more important role than the energy conservation that is not respected by the coalescence model. Therefore, it would be important to clarify the differences between our dynamical approach and the coalescence model.

As we have discussed before, even in the more general coalescence model [Eq. (1)], the momenta of the constituent particles are still nearly aligned to the momentum of the produced particle. In the case of deuteron, this is because the deuteron wave function prefers nearly equal momenta for its constituent nucleons, i.e., $k=0$ in Eq. (2). Combined with the momentum conservation, this leads to $\boldsymbol{p}_{d} / 2 \approx \boldsymbol{p}_{1} \approx \boldsymbol{p}_{2}$, but keeping $\boldsymbol{p}_{d}=\boldsymbol{p}_{1}+\boldsymbol{p}_{2}$. Therefore, although employing the physical deuteron wave function can relax the momentum constraint $\boldsymbol{p}_{d} / 2=\boldsymbol{p}_{1}=\boldsymbol{p}_{2}$, the momenta of the constituent nucleons remain restricted and take only values around the region that appears in the naive coalescence model, which in turn brings the $p_{T}$ dependence of deuteron $v_{2}$ to scale approximately according to its constituent nucleon number.

In the dynamical model, the role of the deuteron wave function appears through the squared transition amplitude for $N N \rightarrow d \pi$, where the energy is conserved. As a result, the allowed momenta of the nucleons forming the outgoing deuteron are different from those in the coalescence model. This can be seen in Fig. 4, which shows the allowed
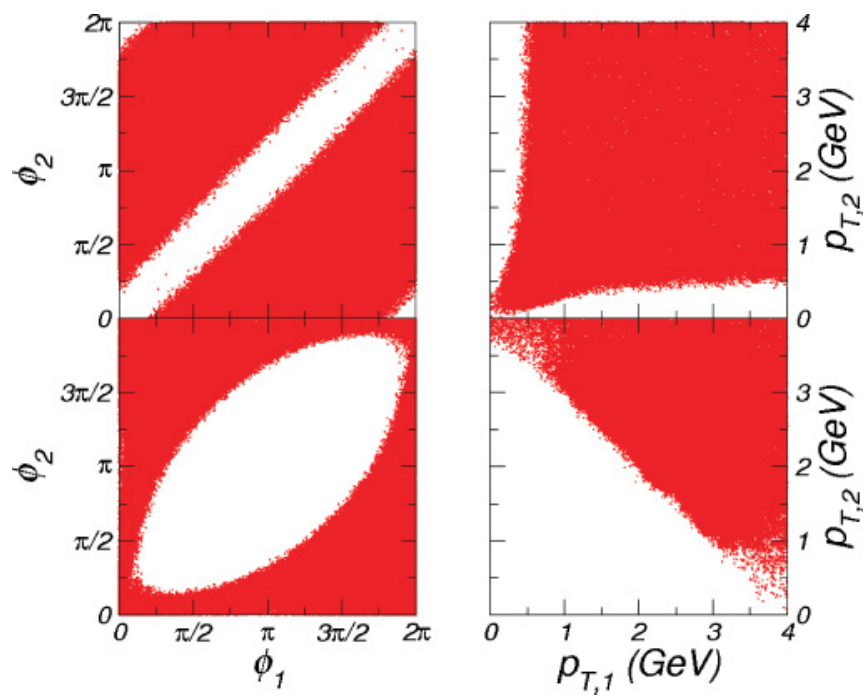

FIG. 4. (Color online) The allowed regions of $\boldsymbol{p}_{1}$ and $\boldsymbol{p}_{2}$ in $N N \rightarrow d \pi$ reaction. The empty space represents the unphysical phase space. Left panels show the phase space in the azimuthal angles $\phi_{1}$ and $\phi_{2}$ of the two nucleons and right panels show the phase space in $\left|\boldsymbol{p}_{T, 1}\right|$ and $\left|\boldsymbol{p}_{T, 2}\right|$. Upper panels are for $p_{T, d}=0.1 \mathrm{GeV}$ and lower panels for $p_{T, d}=4.0 \mathrm{GeV}$. The deuteron azimuthal angle is fixed at $\Phi_{d}=0$. 
momenta $\boldsymbol{p}_{T, 1}$ and $\boldsymbol{p}_{T, 2}$ of nucleons to form a deuteron of $p_{T, d}=0.1 \mathrm{GeV}$ (upper panels) and $4.0 \mathrm{GeV}$ (lower panels) with $\Phi_{d}=0$. The naive coalescence model allows only one point in each figure, namely $\phi_{1}=\phi_{2}=0$ in the left panels and $p_{T, 1}=p_{T, 2}=p_{T, d} / 2$ in the right panels. The more general coalescence model would allow a larger region, but the major contribution comes from the region of $\left(p_{T, 1}, p_{T, 2}\right) \approx\left(p_{T, d} / 2, p_{T, d} / 2\right)$ and $\left(\phi_{1}, \phi_{2}\right) \approx(0,0)$ (modulo $2 \pi)$. The dynamical model based on the $N N \rightarrow d \pi$ reaction covers the phase space shown by the shaded areas in the figure, which may or may not include those involved in the coalescence model. For the phase space in transverse momenta, because of the exponential form of the nucleon $p_{T}$ distribution function, the main contribution in the dynamical approach still comes from that allowed in the more general coalescence model, i.e., $p_{T, 1} \approx p_{T, 2}$. For the phase space in the azimuthal angles, one can find that the region allowed in the dynamical model for $p_{T, d}=4.0 \mathrm{GeV}$ (the lower-left panel of Fig. 4) covers that of the coalescence model, which gives the major contribution to deuteron production. This explains the resemblance of our result with the coalescence model in the intermediate and large $p_{T}$ regions.

The similarity between the two models does not hold, however, in the low $p_{T}$ region. As shown in upper panels of Fig. 4 for $p_{T, d}=0.1 \mathrm{GeV}$, the kinematically allowed phase space in the azimuthal angles is very different from the allowed region in the coalescence model. In fact, the physical phase space excludes the region of $\left(\phi_{1}, \phi_{2}\right) \approx(0,0)$ (modulo $\left.2 \pi\right)$ as a result of energy-momentum conservation and the phase space of the coalescence model is not physically allowed. Therefore, the energy conservation, which is neglected in the coalescence model, plays an important role in the low $p_{T}$ region as one would have expected. In the dynamical model based on the $N N \rightarrow d \pi$ reaction in heavy-ion collisions, there is thus no a priori reason to expect to have the constituent number scaling of the deuteron $v_{2}$ at low $p_{T}$ region.

The scaling behavior of the deuteron $v_{2}$ shown in the low $p_{T}$ region in Fig. 3(b) is a coincidence coming from the parameterized nucleon $v_{2}$ used in the calculation. In fact, if we allow negative $v_{2, N}$ at very low $p_{T}$ region, the coalescence model would predict a negative $v_{2, d}$ as expected from the scaling behavior. However, in the present dynamic model calculation, although inclusion of the radial flow effect as well as deuteron final-state interactions is still required, this expectation is not valid any more in the low $p_{T}$ region, and we always have small but positive $v_{2, d}$ in this region even if we assume negative nucleon $v_{2}$ for low $p_{T}$. This is because of the fact that the nucleon azimuthal distribution function is weighted by the nucleon $p_{T}$ and its distribution function as can be seen in Eq. (10). Therefore, for $p_{T, d}=0.1 \mathrm{GeV}$, the main contribution to deuteron production comes from nucleons of $p_{T} \sim$ $0.5 \mathrm{GeV}$, whereas it must be $\sim 0.05 \mathrm{GeV}$ in the coalescence model due to the constraint on the magnitude of the momenta. Furthermore, the physical azimuthal angles in the dynamical model do not include the region allowed in the coalescence model. Consequently, these cause the main difference between the coalescence model and the dynamical model in the present work.
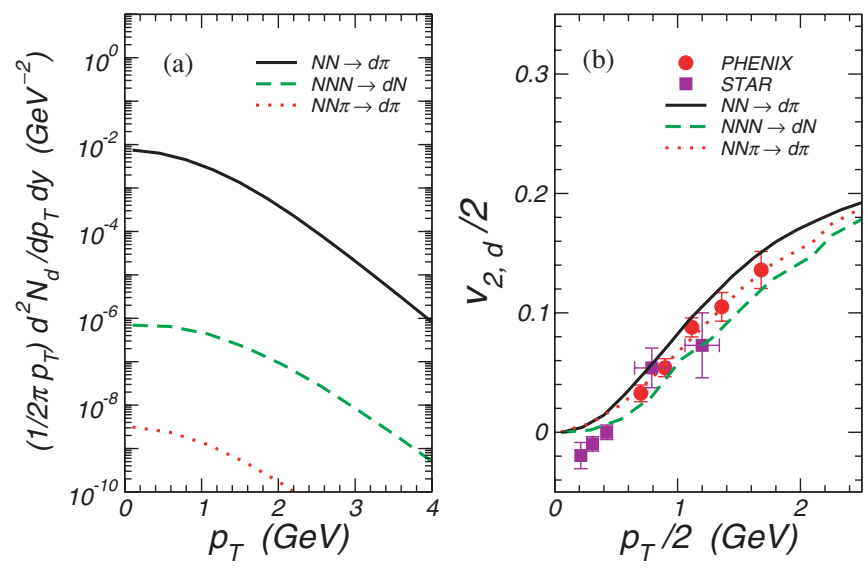

FIG. 5. (Color online) Calculated deuteron $p_{T}$ spectrum (a) and scaled $v_{2}$ (b) at midrapidity from $N N \rightarrow d \pi$ (solid lines), $N N N \rightarrow$ $d N$ (dashed lines), and $N N \pi \rightarrow d \pi$ (dotted lines) reactions. Experimental data are from Refs. $[16,17]$.

\section{B. Deuteron spectrum and elliptic flow from three-body reactions}

For deuteron production from the three-body $N N N \rightarrow$ $d N$ and $N N \pi \rightarrow d \pi$ reactions, the deuteron transversemomentum spectrum and differential elliptic flow can be similarly calculated as in the case of the two-body $N N \rightarrow d \pi$ reaction. Multiplying the calculated rates by the same hadronic matter volume $V \approx 3,700 \mathrm{fm}^{3}$ at freeze out and the time interval $\Delta \tau \approx 4 \mathrm{fm} / c$, the results are shown in Fig. 5 by the dashed and dotted lines, respectively. For comparison, results based on the two-body $N N \rightarrow d \pi$ reaction, which have been shown in Fig. 3, are also given by solid lines.

Although the physically allowed nucleon phase spaces for deuteron production in $N N N$ and $N N \pi$ reactions are different from that in the $N N \rightarrow d \pi$ reaction, the predicted deuteron $v_{2}$ from the three reactions do not show drastic difference from each other as shown in Fig. 5(b), and all lie close to the experimental data except at very low momentum where the deuteron $v_{2}$ is negative in the preliminary data from the STAR Collaboration [17].

In contrast to the deuteron $v_{2}$, the predicted deuteron transverse momentum spectrum depends strongly on the reaction mechanism. As shown in Fig. 5(a), contributions from three-body reactions are suppressed by several orders of magnitude compared to that from the two-body reaction. This is mainly due to the low nucleon and pion densities at freeze-out. Another possible, but subsidiary, reason for this behavior is the magnitude of the reaction amplitudes used in our study. The very simple model we have used for the $N N N \rightarrow d N$ and $N N \pi \rightarrow d \pi$ reactions may have underestimated their contributions to deuteron production. On the contrary to the phase space in $N N \rightarrow d \pi$ reaction (Fig. 4), the phase space in the three-to-two-body reactions has no restrictions on the angles and magnitudes of the momenta of three initial particles. 


\section{SUMMARY AND DISCUSSIONS}

In this article, we studied the deuteron elliptic flow in heavy-ion collisions at RHIC using a dynamical model for deuteron production that conserves both momentum and energy. Because elliptic flows in relativistic heavy-ion collisions are mainly generated during early partonic stage, deuteron elliptic flow is expected to not change much during the hadronic stage, we have considered the deuteron production rate from the $N N \rightarrow d \pi$ reaction at the thermal freeze-out. The deuteron yield is then obtained from multiplying the production rate by the volume of the hadronic matter at freeze-out and a time interval of about $4 \mathrm{fm} / c$. Because of the incomplete treatment of radial flow effect and the neglect of deuteron final-state interactions, the resulting deuteron $p_{T}$ spectrum is somewhat softer than that measured in experiments. The obtained result for the deuteron $v_{2}$ agrees, however, reasonably with the measured one, except at very low $p_{T}$ where the latter is negative. Our results further show that this model can reproduce the predictions from the coalescence model. The origin of this result could be understood from the physically allowed phase space of nucleon momenta in deuteron production. At large $p_{T}$, the phase space in our dynamical model covers that of the coalescence model, which eventually dominate the production process because of the nucleon distribution functions. Therefore, our dynamical model supports the description of the coalescence model. However, at very low $p_{T}$, the nucleon phase space in the coalescence model turns out to be physically forbidden because of energy-momentum conservation. Thus, deuteron production via $N N \rightarrow d \pi$ generally does not reproduce the prediction of the coalescence model at very low $p_{T}$. Although the prediction from the dynamical model follows the scaling behavior, this property is strongly dependent on the nucleon $v_{2}$ at the low $p_{T}$ region.

We have also considered three-body $N N N \rightarrow d N$ and $N N \pi \rightarrow d \pi$ reactions for deuteron production in heavy-ion collisions. Because of the different nucleon phase space involved in these reactions from that in the two-body $N N \rightarrow d \pi$ reaction, these reactions lead to somewhat different predictions on the deuteron $v_{2}$. The deuteron production rates from these reactions are, however, suppressed compared to that from the two-body $N N \rightarrow d \pi$ reaction by several orders of magnitude. This implies that deuteron production in heavy-ion collisions is mainly due to the $N N \rightarrow d \pi$ reaction, which has the largest cross section among the $N N \rightarrow d X$ reactions [23]. We have also found that all models considered in the present study give positive deuteron $v_{2}$ at very low $p_{T}$. This is in contrast to preliminary data from the STAR Collaboration that deuterons produced in heavy-ion collisions at RHIC may have negative $v_{2}$ at very low $p_{T}$ region. To obtain negative deuteron $v_{2}$ required negative nucleon $v_{2}$ in the coalescence model. Therefore, precise measurements of both the nucleon and deuteron $v_{2}$ in this transverse momentum region are highly desirable for understanding the deuteron transverse momentum and elliptic flow in heavy-ion collisions. For our dynamical model approach, there are several issues that deserve further studies. As mentioned in Sec. VI, final-state interactions of deuterons, particularly the $\pi d$ elastic scattering, need to be taken into

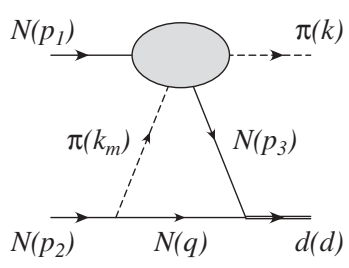

FIG. 6. Diagram for the $N N \rightarrow d \pi$ reaction. The blob denotes the $\pi N \rightarrow \pi N$ elastic scattering.

account via a microscopic transport model or a schematic kinetic model. Also, the deuteron production amplitude used in the present study assumes a pointlike structure of the deuteron. For a more realistic description, the spread of the deuteron wave function should be taken into account in the production amplitude to render a more direct comparison with the coalescence model.

\section{ACKNOWLEDGMENTS}

We are grateful to Wei Liu for useful discussions. This work was supported by the US National Science Foundation under Grant No. PHY-0457265 and the Welch Foundation under Grant No. A-1358.

\section{APPENDIX A: $N \boldsymbol{N} \rightarrow d \pi$ REACTION}

The $N N \rightarrow d \pi$ reaction can be described by the one-pion exchange diagram shown in Fig. 6. In this model, one of the incoming nucleons emits a virtual pion that causes the $\pi N \rightarrow \pi N$ reaction shown by the blob in Fig. 6 before forming the outgoing deuteron. There are other diagrams that include the nucleon exchange diagrams but it is well-known that their contributions are negligible [41]. In this study, we thus adopt a simple model for the $N N \rightarrow d \pi$ reaction of Fig. 6 following the method of Refs. [42,43]. Here we focus on the reaction of $p p \rightarrow d \pi^{+}$and use the isospin relations $\sigma\left(p p \rightarrow d \pi^{+}\right)=\sigma\left(n n \rightarrow d \pi^{-}\right)=2 \sigma\left(p n \rightarrow d \pi^{0}\right)$ to obtain the isospin averaged cross section $\sigma(N N \rightarrow d \pi)=$ $\sigma\left(p p \rightarrow d \pi^{+}\right)$.

Using the momenta indicated in Fig. 6, the amplitude for the $p p \rightarrow d \pi^{+}$reaction can be written as

$$
\begin{aligned}
T= & -\frac{i}{(2 \pi)^{4}} \int d^{4} q \bar{v}\left(p_{2}\right) \Gamma_{\pi N N} \frac{1}{\left(p_{2}-q\right)^{2}-M_{\pi}^{2}} \\
& \times \frac{-q \cdot \gamma+M_{N}}{q^{2}-M_{N}^{2}} \Gamma_{d N N} \frac{(d-q) \cdot \gamma+M_{N}}{(d-q)^{2}-M_{N}^{2}} \\
& \times(A+B k \cdot \gamma) u\left(p_{1}\right),
\end{aligned}
$$

where $M_{N}$ and $M_{\pi}$ are, respectively, the nucleon and pion masses, and $\Gamma_{\pi N N}$ and $\Gamma_{d N N}$ denote, respectively, the pionnucleon and deuteron-nucleon vertices. The functions $A$ and $B$ depend on the Mandelstam variables and are related to the invariant $\pi N$ elastic-scattering amplitude by

$$
\mathcal{M}(\pi N \rightarrow \pi N)=\bar{u}\left(p_{f}\right)(A+B k \cdot \gamma) u\left(p_{i}\right),
$$

where $p_{i}$ and $p_{f}$ are the initial and final nucleon momenta, respectively. The squared matrix element can be written in 
terms of the $\pi N$ elastic-scattering cross section as

$$
|\mathcal{M}(\pi N \rightarrow \pi N)|^{2}=(8 \pi)^{2} 2 s_{\pi N} \frac{d \sigma_{\pi N}}{d \Omega_{\pi N}}\left(s_{\pi N}, u_{\pi N}\right) .
$$

where the Mandelstam variables for the $\pi N$ system are $s_{\pi N}=$ $\left(k_{i}+p_{i}\right)^{2}$ and $u_{\pi N}=\left(k_{i}-p_{f}\right)^{2}$, with $k_{i}$ being the momentum of the initial pion. In our qualitative study, the elastic $\pi N$ scattering can be well approximated by assuming that it is dominated by the $\Delta$ resonance with the interaction Lagrangian

$$
\mathcal{L}_{\pi N \Delta}=\frac{f_{\pi N \Delta}}{M_{\pi}} \bar{\Delta}^{\mu} \partial_{\mu} \pi N+\text { H.c. },
$$

where $f_{\pi N \Delta}=2.23$ and the isospin factors can be found, e.g., in Ref. [44].

Following Ref. [41], the spin-averaged squared amplitude is then given by

$$
\begin{aligned}
|\mathcal{M}|^{2}= & \frac{1}{4} \sum_{\text {spins }}|T|^{2} \\
= & -\left[\frac{\kappa}{M_{d}} \frac{g_{\pi N N} G_{\pi N N}\left(k_{m}^{2}\right)}{k_{m}^{2}-M_{\pi}^{2}}\right]^{2}\left[\frac{g_{d N N}}{M_{N}} \frac{1}{2 \sqrt{2} M_{d}}\right]^{2} \\
& \times \frac{1}{2} k_{m}^{2} M_{d}^{2}\left(2 M_{N}+M_{d}\right)^{2} \\
& \times\left[3\left(1+\frac{\varrho}{\sqrt{2}}\right)^{2}-\frac{6 \varrho}{\sqrt{2}}\left(1+\frac{\varrho}{\sqrt{2}}\right)+\left(\frac{3 \varrho}{\sqrt{2}}\right)^{2}\right] \\
& \times|\mathcal{M}(\pi N \rightarrow \pi N)|^{2} .
\end{aligned}
$$

In the above, $\varrho(\approx 4 \%)$ is the amount of $D$-wave state in the deuteron wave function, $M_{d}$ is the deuteron mass, and $\kappa \equiv$ $\sqrt{M_{N} \varepsilon}$ with the deuteron binding energy $\varepsilon(\approx 2.23 \mathrm{MeV})$. The pion-nucleon coupling constant is denoted by $g_{\pi N N}$ with a value $g_{\pi N N}^{2} / 4 \pi=14.0$, and $G_{\pi N N}\left(q^{2}\right)$ is the form factor

$$
G_{\pi N N}\left(q^{2}\right)=\frac{\Lambda^{2}-M_{\pi}^{2}}{\Lambda^{2}-q^{2}}
$$

with $\Lambda=0.73 \mathrm{GeV}$. The deuteron-nucleon coupling constant $g_{d N N}$ is given by

$$
g_{d N N}=\left[\frac{16 \pi \kappa}{M_{N}\left(1-r_{t} \kappa\right)\left(1+\varrho^{2}\right)}\right]^{1 / 2},
$$

where $r_{t}(\approx 1.75 \mathrm{fm})$ is the effective range for the spintriplet nucleon-nucleon elastic scattering. To reproduce the experimental total cross section for $p p \rightarrow d \pi^{+}$within this simple model, we use $M_{\Delta}=1200 \mathrm{MeV}$. The calculated cross section is shown in Fig. 7 and is seen to reproduce the measured one very well.

\section{APPENDIX B: $N N N \rightarrow d N$ AND $N N \pi \rightarrow d \pi$ REACTIONS}

The amplitudes for producing deuterons from three-body reactions $N N N \rightarrow d N$ and $N N \pi \rightarrow d \pi$ are approximated by making use of the amplitude of $N N \rightarrow d \pi$ obtained above. For the $N N N \rightarrow d N$ reaction, we consider the one-pion exchange process, namely the collision of two initial nucleons gives the deuteron and an off-shell pion, which is then absorbed by the spectator nucleon. In this approximation, the reaction

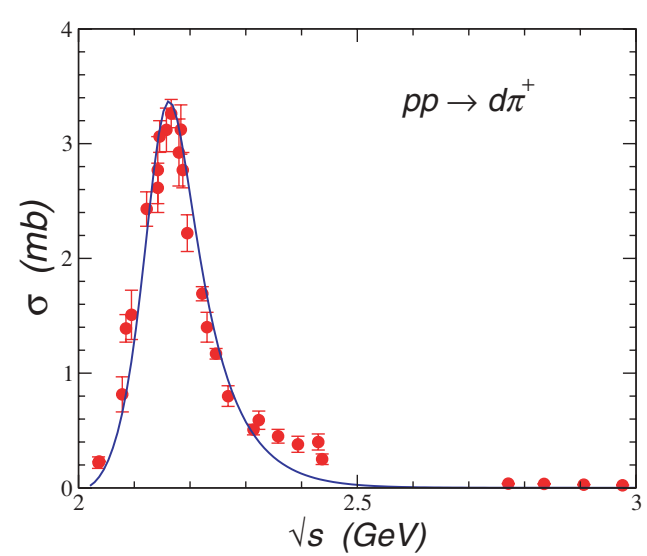

FIG. 7. (Color online) Total cross section for the $p p \rightarrow d \pi^{+}$ reaction. The experimental data are from Refs. [45-48].

amplitude becomes

$$
\begin{aligned}
& |\mathcal{M}(N N N \rightarrow d N)|^{2} \\
& \quad=\frac{4 G_{\pi N N}^{2}}{\left(q_{m}^{2}-M_{\pi}^{2}\right)^{2}}\left(p_{3} \cdot p_{f}-M_{N}^{2}\right)|\mathcal{M}(N N \rightarrow d \pi)|^{2},
\end{aligned}
$$

where $q_{m}$ is the intermediate pion momentum, $p_{3}$ is the momentum of the initial spectator nucleon, and $p_{f}$ is the nucleon momentum in the final state.

Replacing the off-shell pion by an off-shell $\rho$ meson, which is then absorbed by a spectator pion leads to an approximate description of the $N N \pi \rightarrow d \pi$ reaction. The amplitude for the subprocess $N N \rightarrow d \rho$ can be obtained by the same method of previous section for obtaining the $N N \rightarrow d \pi$ amplitude. The result is thus similar to Eq. (A5) with $|\mathcal{M}(\pi N \rightarrow \pi N)|^{2}$ replaced by $|\mathcal{M}(\pi N \rightarrow \rho N)|^{2} / 2$. A complete analysis of the $\pi N \rightarrow \rho N$ reaction requires many possible terms arising from meson exchanges and nucleon resonance contributions. For a simple estimate on the cross section for this reaction, we describe it by one-pion exchange only and fit the experimental data with suitable form factors.
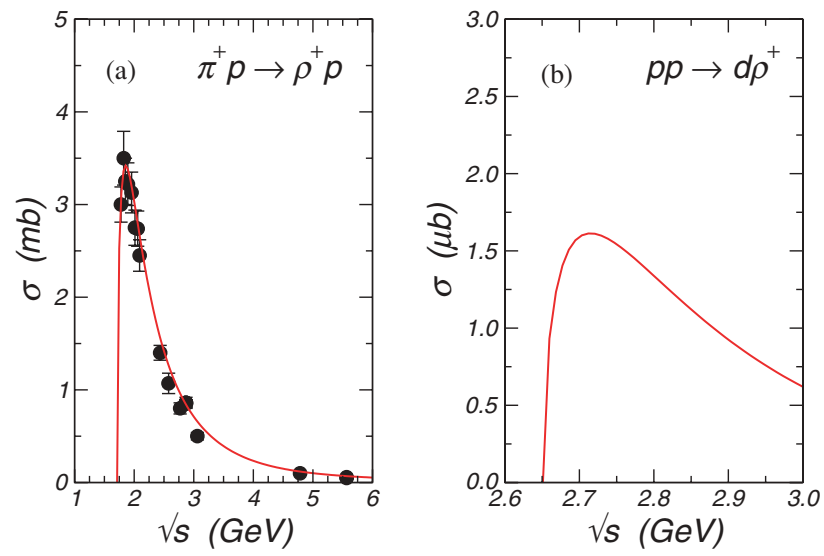

FIG. 8. (Color online) Total cross sections for $\pi^{+} p \rightarrow \rho^{+} p$ (a) and $p p \rightarrow d \rho^{+}$(b). Experimental data are from Ref. [49]. 
The relevant effective Lagrangians are

$$
\begin{aligned}
\mathcal{L}_{\rho \pi \pi} & =g_{\rho \pi \pi} \rho^{\mu} \cdot\left(\pi \times \partial_{\mu} \pi\right), \\
\mathcal{L}_{\pi N N} & =-\frac{g_{\pi N N}}{2 M_{N}} \bar{N} \tau \cdot \partial_{\mu} \pi \gamma_{5} \gamma^{\mu} N,
\end{aligned}
$$

where $g_{\rho \pi \pi}=6.02$ and $g_{\pi N N}^{2} /(4 \pi)=14.0$. The form factor in the form of $\Lambda^{4} /\left[\Lambda^{4}+\left(t-M_{\pi}^{2}\right)^{2}\right]$ is used with
$\Lambda=0.65 \mathrm{GeV}$. The $N N \pi \rightarrow d \pi$ amplitude is then given by

$$
\begin{aligned}
& |\mathcal{M}(N N \pi \rightarrow d \pi)|^{2} \\
& \quad=\frac{2 G_{\rho N N}^{2}}{\left(q_{m}^{2}-M_{\rho}^{2}\right)^{2}}\left(p_{3} \cdot p_{f}-M_{\pi}^{2}\right)|\mathcal{M}(N N \rightarrow d \rho)|^{2} .
\end{aligned}
$$

Our results on the total cross sections for $\pi^{+} p \rightarrow \rho^{+} p$ and $p p \rightarrow d \rho^{+}$are shown in Fig. 8. The former is seen to agree with the measured cross section.
[1] J.-Y. Ollitrault, Phys. Rev. D 46, 229 (1992).

[2] S. Voloshin and Y. Zhang, Z. Phys. C 70, 665 (1996).

[3] W. Reisdorf and H. G. Ritter, Annu. Rev. Nucl. Part. Sci. 47, 663 (1997).

[4] P. Danielewicz, R. A. Lacey, P. B. Gossiaux, C. Pinkenburg, P. Chung, J. M. Alexander, and R. L. McGrath, Phys. Rev. Lett. 81, 2438 (1998).

[5] R. A. Lacey, Nucl. Phys. A774, 199 (2006).

[6] STAR Collaboration, J. Adams et al., Nucl. Phys. A757, 102 (2005).

[7] PHENIX Collaboration, K. Adcox et al., Nucl. Phys. A757, 184 (2005).

[8] P. F. Kolb, J. Sollfrank, and U. Heinz, Phys. Rev. C 62, 054909 (2000).

[9] Z. W. Lin and C. M. Ko, Phys. Rev. C 65, 034904 (2002).

[10] V. Greco, C. M. Ko, and P. Lévai, Phys. Rev. Lett. 90, 202302 (2003); Phys. Rev. C 68, 034904 (2003).

[11] R. C. Hwa and C. B. Yang, Phys. Rev. C 67, 034902 (2003); 67, 064902 (2003).

[12] R. J. Fries, B. Müller, C. Nonaka, and S. A. Bass, Phys. Rev. Lett. 90, 202303 (2003); Phys. Rev. C 68, 044902 (2003).

[13] D. Molnár and S. A. Voloshin, Phys. Rev. Lett. 91, 092301 (2003).

[14] P. F. Kolb, L.-W. Chen, V. Greco, and C. M. Ko, Phys. Rev. C 69, 051901(R) (2004).

[15] PHENIX Collaboration, S. Adare et al., Phys. Rev. Lett. 98, 162301 (2007).

[16] PHENIX Collaboration, S. Afanasiev et al., Phys. Rev. Lett. 99, 052301 (2007).

[17] H. Liu (STAR Collaboration), J. Phys. G 34, S1087 (2007).

[18] J. I. Kapusta, Phys. Rev. C 21, 1301 (1980); C. B. Dover, U. Heinz, E. Schnedermann, and J. Zimányi, Phys. Rev. C 44, 1636 (1991).

[19] L. McLerran, J. Phys. G 34, S583 (2007).

[20] L. Ravagli and R. Rapp, Phys. Lett. B655, 126 (2007).

[21] Z. W. Lin, C. M. Ko, B. A. Li, B. Zhang, and S. Pal, Phys. Rev. C 72, 064901 (2005).

[22] L. W. Chen, V. Greco, C. M. Ko, S. H. Lee, and W. Liu, Phys. Lett. B601, 34 (2004).

[23] H. L. Anderson et al., Phys. Rev. Lett. 26, 108 (1971).

[24] R. Mattiello, A. Jahns, H. Sorge, H. Stöcker, and W. Greiner, Phys. Rev. Lett. 74, 2180 (1995); R. Mattiello, H. Sorge, H. Stöcker, and W. Greiner, Phys. Rev. C 55, 1443 (1997).
[25] L.-W. Chen, C. M. Ko, and B. A. Li, Phys. Rev. C 68, 017601 (2003).

[26] L.-W. Chen, C. M. Ko, and B.-A. Li, Nucl. Phys. A729, 809 (2003).

[27] T. Z. Yan et al., Phys. Lett. B638, 50 (2006).

[28] L. P. Csernai and J. I. Kapusta, Phys. Rep. 131, 223 (1986).

[29] P. Danielewicz and G. F. Bertsch, Nucl. Phys. A533, 712 (1991).

[30] A. Deloff and T. Siemiarczuk, Nucl. Phys. A555, 659 (1993).

[31] D. E. Gonzalez Trotter et al., Phys. Rev. C 73, 034001 (2006).

[32] PHENIX Collaboration, S. S. Adler et al., Phys. Rev. C 69, 034909 (2004).

[33] R. A. Fisher and L. H. C. Tippett, Proc. Cambridge Philos. Soc. 24, 180 (1928); E. J. Gumbel, Statistics of Extremes (Columbia University Press, New York, 1958).

[34] V. Greco and C. M. Ko, Phys. Rev. C 70, 024901 (2004).

[35] P. F. Kolb, P. Huovinen, U. Heinz, and H. Heiselberg, Phys. Lett. B500, 232 (2001).

[36] P. Huovinen et al., Phys. Lett. B503, 58 (2001).

[37] X. Dong et al., Phys. Lett. B597, 328 (2004).

[38] PHENIX Collaboration, S. S. Adler et al., Phys. Rev. Lett. 94, 122302 (2005)

[39] M. A. Khandaker, M. Doss, I. Halpern, T. Murakami, D. W. Storm, D. R. Tieger, and W. J. Burger, Phys. Rev. C 44, 24 (1991).

[40] B. A. Li and C. M. Ko, Phys. Rev. C 52, 2037 (1995); B. A. Li, A. T. Sustich, B. Zhang, and C. M. Ko, Int. J. Mod. Phys. E 10, 267 (2001).

[41] W. Grein et al., Ann. Phys. (NY) 153, 301 (1984).

[42] T. Yao, Phys. Rev. 134, B454 (1964).

[43] G. W. Barry, Ann. Phys. (NY) 73, 482 (1972).

[44] Y. Oh, K. Nakayama, and T.-S. H. Lee, Phys. Rep. 423, 49 (2006).

[45] H. L. Anderson et al., Phys. Rev. D 9, 580 (1974).

[46] F. Shimizu et al., Nucl. Phys. A386, 571 (1982).

[47] GEM Collaboration, M. Betigeri et al., Phys. Rev. C 63, 044011 (2001).

[48] COSY-TOF Collaboration, S. Abd El-Samad et al., Eur. Phys. J. A 30, 443 (2006).

[49] Y. Williamson et al., Phys. Rev. Lett. 29, 1353 (1972); Reaction Data Database, http://durpdg.dur.ac.uk/HEPDATA/REAC. 